\title{
A novel and efficient session spanning biometric and password based three- factor authentication protocol for consumer USB mass storage devices
}

Article

Accepted Version

Giri, D., Sherratt, S. and Maitra, T. (2016) A novel and efficient session spanning biometric and password based three-factor authentication protocol for consumer USB mass storage devices. IEEE Transactions on Consumer Electronics, 62 (3). pp. 283-291. ISSN 0098-3063 doi:

https://doi.org/10.1109/TCE.2016.7613195 Available at https://centaur.reading.ac.uk/67912/

It is advisable to refer to the publisher's version if you intend to cite from the work. See Guidance on citing.

Published version at: http://ieeexplore.ieee.org/document/7613195

To link to this article DOI: http://dx.doi.org/10.1109/TCE.2016.7613195

Publisher: IEEE

All outputs in CentAUR are protected by Intellectual Property Rights law, including copyright law. Copyright and IPR is retained by the creators or other copyright holders. Terms and conditions for use of this material are defined in the End User Agreement. 


\section{www.reading.ac.uk/centaur}

\section{CentAUR}

Central Archive at the University of Reading

Reading's research outputs online 


\title{
Full-Text version
}

Title: $\quad$ A Novel and Efficient Session Spanning Biometric and Password based Three-factor Authentication Protocol for Consumer USB Mass Storage Devices

Authors: Debasis Giri,

Department of Computer Science and Engineering, Haldia Institute of Technology, Haldia721657, India

(e-mail: debasis giri@hotmail.com)

R. Simon Sherratt, Fellow, IEEE

Department of Biomedical Engineering, the University of Reading, RG6 6AY,

UK

(e-mail: sherratt@ieee.org)

Tanmoy Maitra

Department of Computer Science and Engineering, Jadavpur University, Kolkata-700032, India

(e-mail: tanmoy.maitra.in@ieee.org)

Publication: IEEE Transactions on Consumer Electronics

Volume: $\quad 62$

Issue: $\quad 3$

pp.: $\quad 283-291$

Date: $\quad$ August 2016

DOI: $\quad \underline{10.1109 / T C E .2016 .7613195}$

\begin{abstract}
This paper proposes a key agreement scheme after secure authentication to prevent the unauthorized access of the data stored in a Universal Serial Bus (USB) Mass Storage Device (MSD). Due to the system architecture of this proposed scheme, authorized users can store their data in a secure encrypted form after performing authentication. The novelty of this work is that users can retrieve the encrypted data in not only the current session but also across different sessions, thus reducing the required communications overhead. This paper then analyses the security of the proposed protocol through a formal analysis to demonstrate that the information has been stored securely and is also protected offering strong resilience to relevant security attacks. The computational and communication costs of the proposed scheme is analyzed and compared to related works to show that the proposed scheme has an improved tradeoff for computational cost, communication cost and security.
\end{abstract}

\section{Index Terms}

Biometrics, Consumer Storage, Mass Storage Device, Password, USB, FLASH. 


\section{INTRODUCTION}

Universal Serial Bus (USB) is a well-accepted ubiquitous serial interface. It is commonly used to connect peripheral devices such as keyboards, mice, cell phones, printers, scanner, Mass Storage Devices (MSD), etc. to a host Personal Computer (PC). The high availability, high data transfer rate and ease of connectivity are primary advantages of USB. However, at an application level, MSD's suffer from significant security weaknesses such as (1) an unauthorized user could easily read or steal confidential information as the information is stored in plaintext form, and (2) an attacker could intercept all the information sent over the bus as the channel between device and the host computer USB port can be open to the attacker (e.g. physical, virus or malware). User authentication and session key agreement are designed in such a way that they can resolve the aforementioned difficulties.

Many authors have proposed authentication schemes. In 2004, Ku and Chen [1] proposed an authentication scheme which was solely based on passwords, but Yoon, Ryu and Yoo [2] found that Ku and Chen's scheme [1] was vulnerable to the parallel session attack and so they proposed an enhanced authentication scheme. In 2010, Yang, Wu and Chiu [3] also proposed a password based protocol for USB MSDs. Research then focused on complementing passwords with user biometrics [4] to offer a further factor to the authentication protocol in order to provide improved security. In 2013, Lee, Chen and Wu [5] proposed a biometric based three-factor authentication protocol for USB MSDs. However, in 2014, He et al. [6] showed that Lee, Chen and Wu's scheme [5] could not resist the password guessing attack, the Denial of Service (DoS) attack and the replay attack, so He et al. [6] proposed a biometrics based three-factor security protocol for USB MSDs. In 2015, Giri et al. [7] also proposed an authentication scheme for USB MSDs using biometrics and password protection, where after performing mutual authentication, a session key was established to encrypt user's data.

This research has identified that there are two drawbacks in the previously presented schemes, (1) each time a user sends a request to the authentication server to decrypt a stored file the server sends the same part of the key. If an authorized user stores that part of the key then it is no longer needed to send a subsequent request to the server to obtain the same part of key at a later date. However, in security protocols for consumer USB MSDs it is required to authenticate each and every time a user wants to store and retrieve the file; (2) if an authorized user wishes to retrieve a stored encrypted file within the same login session as when the file was originally stored into the memory of USB device, then the user has to perform all the authentication steps (mainly login phase) which unfortunately increases the computational and communication costs, and reduces device usability.

This research presents a system architecture for creating a Three-factor Security Protocol (TSP), where authorized users can store their files in encrypted form and not only can retrieve the original file in the current session but additionally in subsequent sessions after performing the authentication procedure respectively.

The rest of the paper is organized as follows: Section II presents the required mathematical concepts. Section III introduce the proposed scheme by describing the system architecture. Section IV presents the authentication scheme proposed in this work and the security of the proposed scheme is analyzed in Section V. Section VI presents the performance evaluation and Section VII concludes the paper. Table I shows the nomenclature that is used throughout the paper. 
TABLE I

NOMENCLATURE

\begin{tabular}{ll}
\hline \hline Term & Usage \\
\hline$U_{i}$ & i-th user \\
$A S$ & Remote authentication server \\
$x$ & Secret key of authentication server $A S$ \\
$p w_{i}$ & Password of user $U_{i}$ \\
$B_{i}$ & Biometric parameter of user $U_{i}$ \\
$I D_{i}$ & Identity of user $U_{i}$ \\
$E N C_{k}[]$ & Symmetric key encryption by a key $k$ \\
$D E C_{k}[]$ & Symmetric key decryption by a key $k$ \\
$S K_{i}$ & Shared secret session key between user $U_{i}$ and server $A S$ \\
$\alpha_{i}$ and $\delta_{i}$ & Random numbers generated by $U S B$ device \\
$\beta_{i}$ and $\gamma_{i}$ & Random numbers generated by server $A S$ \\
$F_{i}$ & Unique identity or index of a file \\
$F_{\text {name }}$ & File name \\
$d e s(\cdot)$ & Difference measurement function \\
$d$ & Threshold value \\
$r$ & Integer, first $r$ number of bits of a file \\
$n$ & Integer, total number of bits of a file \\
$T$ & Current timestamp \\
$\Delta T$ & Estimated time delay \\
$h(\cdot)$ & Cryptographic one-way hash function \\
$\oplus$ & Bitwise xor operation \\
$\|$ & Concatenation operation \\
\hline
\end{tabular}

\section{BACKGROUND}

This section defines the collision resistant cryptographic one-way hash function [7], the collision resistant fuzzy extractor [7], [8] and collision resistant secure encryption/decryption technique [9], [10] in order to analyze the security of this proposed scheme.

Definition 1: A collision resistant cryptographic one-way hash function maps a binary string of an arbitrary length to a binary string of fixed length called the hashed value. This paper considers the cryptographic oneway hash function as previously defined [7].

Definition 2: A collision resistant fuzzy extractor can be modeled as two procedures: 1) GEN, which takes a binary string as input, and generates two strings, namely, arbitrary string of length $l$ bit and auxiliary string of length $r$-bit; 2) REP, which takes a binary string and an auxiliary string, and produces an arbitrary string. Furthermore, this paper also defines the same definition of collision resistant fuzzy extractor as previously defined [7].

Definition 3: A secret key is used to encrypt plaintext into ciphertext and the same key is used to decrypt the ciphertext using the symmetric key block encryption/decryption technique. This process can be symbolized as: $C \leftarrow E N C_{k}[M]$ and $M \leftarrow D E C_{k}[C]$, where $M=\{0,1\}^{*}, C=\{0,1\}^{*}$ and $k=\{0,1\}^{n}$, given plaintext $\mathrm{M}$ and ciphertext $\mathrm{C}$ being binary strings of arbitrary length, secret key $\mathrm{k}$ being a binary string of fixed length $n$ and ENC/DEC are encryption and decryption algorithms respectfully. If $A d v_{A}^{E N C / D E C}\left(t_{3}^{1}\right)$ is the advantage to an adversary $A$ to choose a key $k \in_{R}\{0,1\}^{n}$ randomly such that $M=D E C_{k}\left[E N C_{k}[M]\right]$ or $C=E N C_{k}\left[D E C_{k}[C]\right]$ 
for the time duration $t_{3}^{1}$, it can be considered that $A d v_{A}^{E N C / D E C}\left(t_{3}^{1}\right)$ is the advantage computed over random choices made by adversary $A$ for time duration $t_{3}^{1}$. Then the symmetric key encryption/decryption ENC/DEC can be called secure if $A d v_{A}^{E N C / D E C}\left(t_{3}^{1}\right) \leq \xi_{3}^{1}$, for any small $\xi_{3}^{1}>0 . A d v_{A}^{E N C / D E C}\left(t_{3}^{1}\right)$ is represented as:

$$
\operatorname{Adv}_{A}^{E N C I D E C}\left(t_{3}^{1}\right)=\operatorname{Pr}\left[\begin{array}{rl}
k \in_{R}\{0,1\}^{n} \mid C & =E N C_{k}\left[D E C_{k}[C]\right] \vee \\
M & =D E C_{k}\left[E N C_{k}[M]\right]
\end{array}\right]
$$

The symmetric key encryption/decryption algorithm also follows the property of collision resistance as described in Definition 1. If $A d v_{A}^{E N C / D E C}\left(t_{3}^{2}\right)$ is the advantage to $A$ to choose a pair $\left(M, M^{\prime}\right) \in_{R}\{0,1\}^{*} \times\{0,1\}^{*}$ randomly such that $\operatorname{ENC}_{k}[M]=E N C_{k}\left[M^{\prime}\right]$ where $M \neq M^{\prime}$ or $\left(C, C^{\prime}\right) \in_{R}\{0,1\}^{n} \times\{0,1\}^{n}$ such that $D E C_{k}[C]=D E C_{k}\left[C^{\prime}\right]$ where $C \neq C^{\prime}$ for the time duration $t_{3}^{2}$, it can be considered that $A d v_{A}^{E N C / D E C}\left(t_{3}^{2}\right)$ is the advantage computed over random choices made by $A$ for time duration $t_{3}^{2}$. Then the symmetric key encryption/decryption $E N C / D E C$ is called collision resistant if $A d v_{A}^{E N C / D E C}\left(t_{3}^{2}\right) \leq \xi_{3}^{2}$, for any small $\xi_{3}^{2}>0$. $A d v_{A}^{E N C / D E C}\left(t_{3}^{2}\right)$ is represented as:



$A d v_{A}^{E N C / D E C}\left(t_{3}\right)$ is the advantage to $A$ for the time duration $t_{3}$, given by:

$A d v_{A}^{E N C / D E C}\left(t_{3}\right)=\max _{A}\left\{A d v_{A}^{E N C / D E C}\left(t_{3}^{1}\right), A d v_{A}^{E N C / D E C}\left(t_{3}^{2}\right)\right\}$

Then the symmetric key encryption/decryption $E N C / D E C$ is called collision resistant and secure, if $\operatorname{Adv}_{A}^{E N C / D E C}\left(t_{3}\right) \leq \xi_{3}$, for any small $\xi_{3}>0$. 


\section{Architecture}

This section presents the details of the system architecture of the proposed scheme.

At first, user $U_{i}$ registers with an authentication server, $A S$, in order to request authorized access to the USB MSD as shown in Fig. 1(a). $A S$ has the responsibility to verify the legitimacy of $U_{i}$ when $U_{i}$ wishes to read or write a file to a storage device via the USB interface. $U_{i}$ enters their identity, password and biometric parameters to verify legitimacy. Fig. 1(b) shows the communication procedure when $U_{i}$ wants to store a new file into the USB MSD in an encrypted form or wants to decrypt an already stored encrypted file in the USB device.

This research has considered two conditions (1) $U_{i}$ can store a new file in an encrypted form after performing mutual authentication between $U_{i}$ and $A S$ in session $S_{i}$ and can decrypt the newly stored encrypted file within the session $S_{i}$ after obtaining permission from $A S$, (2) $U_{i}$ can decrypt the stored encrypted file within a different session, $S_{j}$, after performing mutual authentication between $U_{i}$ and $A S$. Moreover in this proposed system architecture, the authentication plays a vital role whenever the users try to store/extract a file to/from their USB MSD, each and every time the users need to be authenticated to $A S$.

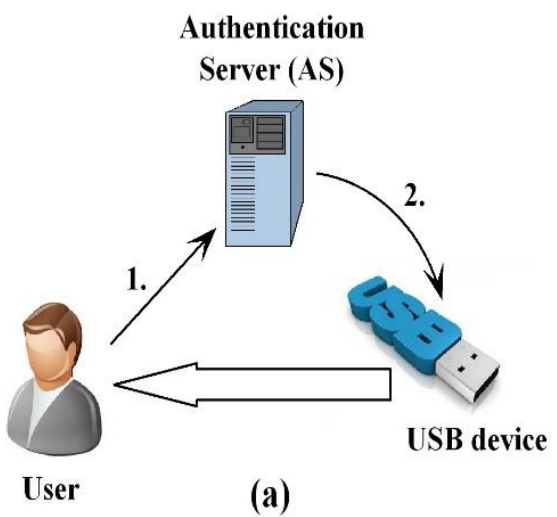

Fig. 1. System architecture of the proposed scheme

(a) Registration procedure (b) File encryption/decryption and authentication procedure.

\section{Proposed SCHEMe From THIS ReSEARCH}

The proposed scheme consists of (1) registration phase, (2) authentication with file encryption and decryption phase in current session, (3) authentication with file decryption phase in different session and (4) password update phase.

1) AS selects a cryptographic one-way hash function $h(\cdot)$. This process can be symbolized as $h:\{0,1\}^{*} \rightarrow\{0,1\}^{l}$, where $l$ is a fixed length (128 bits) integer.

2) $A S$ also selects a symmetric key encryption /decryption algorithm ENC/DEC and a secret key $x$. AS then publishes $\langle E N C / D E C, h(\cdot)\rangle$ as the public parameters while keeping $x$ secret. 


\section{A. Registration Phase}

In the registration phase, $U_{i}$ and $A S$ perform the following steps to obtain authorized information from the USB MSD:

1) $U_{i}$ inputs their biometric parameter $B_{i}$ through a suitable biometric device (e.g. fingerprint reader) to calculate $\left(\psi_{i}, \theta_{i}\right) \leftarrow G E N\left(B_{i}\right) . U_{i}$ also provides their password $p w_{i}$ and identity $I D_{i}$. $U_{i}$ then computes $p w r_{i}=h\left(p w_{i} \| \psi_{i}\right)$ and submits $\left\langle I D_{i}, p w r_{i}\right\rangle$ to $A S$.

2) After receiving $\left\langle I D_{i}, p w r_{i}\right\rangle, A S$ computes $A_{i}=h\left(I D_{i} \| x\right) \oplus p w r_{i}$ and $C_{i}=h\left(h\left(I D_{i} \| x\right) \| p w r_{i}\right)$. AS then stores $\left\langle A_{i}, C_{i}, \operatorname{des}(\cdot)\right\rangle$ in $U_{i}$ 's USB MSD and delivers it to $U_{i}$ securely.

3) After getting the authorized information for the USB MSD, $U_{i}$ computes $K_{i}=\theta_{i} \oplus h\left(I D_{i} \| p w_{i}\right)$, $G_{i}=B_{i} \oplus h\left(I D_{i} \| p w_{i}\right)$ and stores $\left\langle K_{i}, G_{i}\right\rangle$ into the USB MSD. Finally, the USB MSD contains the parameters $\left\langle A_{i}, C_{i}, \operatorname{des}(\cdot), K_{i}, G_{i}\right\rangle$.

\section{B. Authentication with File Encryption and Decryption Phase in the Current Session}

When $U_{i}$ wants to store a new file (larger than $r$ bits) in the USB MSD in an encrypted format, the following steps are executed between $U_{i}$ and $A S$ :

1) $U_{i}$ inserts their USB MSD into the client USB port and inputs their password $p w_{i}$, identity $I D_{i}$ and biometric parameter $B_{i}$. The MSD then computes $B_{i}=G_{i} \oplus h\left(I D_{i} \| p w_{i}\right)$ and then checks condition $\operatorname{des}\left(B_{i}, B_{i}^{\prime}\right) \leq d$. If false, the device rejects $U_{i}$; otherwise the device computes $\theta_{i}=K_{i} \oplus h\left(I D_{i} \| p w_{i}\right)$, $\psi_{i}=\operatorname{REP}\left(B_{i}, \theta_{i}\right)$ and $L_{i}\left(=h\left(I D_{i} \| x\right)\right)=A_{i} \oplus h\left(p w_{i} \| \psi_{i}\right)$. Then the device checks $C_{i}=h\left(L_{i} \| h\left(p w_{i} \| \psi_{i}\right)\right)$ . If the equality fails then the device rejects $U_{i}$; otherwise the device executes the next step.

2) The USB MSD generates random number $\alpha_{i}$, and computes $Q_{i}=E N C_{h\left(I D_{i} \| \psi_{i}\right)}\left[\right.$ File $\left._{\text {rbits }}\right] \oplus \alpha_{i}$, $Z_{i}=L_{i} \oplus \alpha_{i}$ and $W_{i}=h\left(L_{i}\left\|I D_{i}\right\| \alpha_{i}\left\|F_{i}\right\| T_{i}\right)$, where $r, T_{i}$ and $F_{i}$ are the first $r$ number of bits of the file, the current timestamp of $U_{i}$ and index or identity of the file respectively. Finally, message $M S G 1=\left\langle I D_{i}, F_{i}, Q_{i}, Z_{i}, W_{i}, T_{i}\right\rangle$ is sent to $A S$.

3) After receiving $M S G 1$ at timestamp $T_{s}, A S$ checks the format of $I D_{i}$ and condition $\left(T_{s}-T_{i}\right) \leq \Delta T$. If any are invalid $A S$ rejects the message; otherwise $A S$ computes $L_{i}^{\prime}=h\left(I D_{i} \| x\right)$ and $\alpha_{i}^{\prime}=L_{i}^{\prime} \oplus Z_{i}$. Then $A S$ checks $W_{i}=h\left(L_{i}^{\prime}\left\|I D_{i}\right\| \alpha_{i}^{\prime}\left\|F_{i}\right\| T_{i}\right)$. If the equality does not hold, $A S$ rejects the message; otherwise $A S$ generates random number $\beta_{i}$ and computes $X_{i}\left(=E N C_{h\left(I D_{i} \| \psi_{i}\right)}\left[\right.\right.$ file $\left.\left._{r b i t s}\right]\right)=Q_{i} \oplus \alpha_{i}^{\prime}, \quad Y_{i}=\beta_{i} \oplus L_{i}^{\prime}$, $S K_{i}=h\left(\alpha_{i}^{\prime}\left\|\beta_{i}\right\| L_{i}^{\prime} \| T_{S}\right), H_{i}=h\left(x \| F_{i}\right) \oplus S K_{i}, E_{i}=E N C_{x}\left[X_{i} \| F_{i}\right] \oplus S K_{i}$ and

$D_{i}=h\left(I D_{i}\left\|\beta_{i}\right\| \alpha_{i}^{\prime}\left\|T_{s}\right\| S K_{i}\left\|h\left(x \| F_{i}\right)\right\| E N C_{x}\left[X_{i} \| F_{i}\right]\right)$. AS then sends the message $M S G 2=\left\langle E_{i}, D_{i}, Y_{i}, T_{s}, H_{i}\right\rangle$ to $U_{i}$.

4) After receiving $M S G 2$ at timestamp $T_{j}, U_{i}$ checks condition $\left(T_{j}-T_{S}\right) \leq \Delta T$. If invalid $U_{i}$ terminates the session; otherwise $U_{i}$ computes $\beta_{i}^{\prime}=L_{i} \oplus Y_{i}, \quad S K_{i}^{\prime}=h\left(\alpha_{i}\left\|\beta_{i}^{\prime}\right\| L_{i} \| T_{S}\right), \quad\left[h\left(x \| F_{i}\right)\right]^{\prime}=H_{i} \oplus S K_{i}^{\prime} \quad$ and $\left[E n c_{x}\left[X_{i} \| F_{i}\right]\right]^{\prime}=E_{i} \oplus S K_{i}^{\prime}$. Then $U_{i}$ checks $D_{i}=h\left(I D_{i}\left\|\beta_{i}^{\prime}\right\| \alpha_{i}\left\|T_{s}\right\| S K_{i}^{\prime}\left\|\left[h\left(x \| F_{i}\right)\right]^{\prime}\right\|\left[E n c_{x}\left[X_{i} \| F_{i}\right]\right]\right)$. If the equality fails then $U_{i}$ terminates the session; otherwise $U_{i}$ selects a suitable file name $F_{\text {name }}$ for the file and stores the encrypted file as $\left\langle E n c_{x}\left[X_{i} \| F_{i}\right], F_{i}, F_{\text {name }}, E n c_{h\left(x \| F_{i}\right)}\left[\right.\right.$ File $\left.\left._{(n-r) b i t s}\right]\right\rangle$ into the memory of the USB MSD, where (n-r) bits are the remaining bits of the file. Note that if $U_{i}$ maintains a database for 
the file plus key combinations then $U_{i}$ has to also communicate with $A S$ to extract the first $r$ bits of the file. If $r$ or $(n-r)$ bits are larger than key size $k$ then $U_{i}$ breaks $r$ or $(n-r)$ bits into blocks of size equal to the key size $k$ (here 128 bits) and encrypt each block with the counter (CTR) mode [10] of operation to produce the corresponding ciphertext. Plaintext can be retrieved by the same procedure reversely.

When $U_{i}$ wants to decrypt that encrypted file from the USB MSD in the current session, the following steps are executed between $U_{i}$ and $A S$ :

5) $U_{i}$ sends message $\left\langle E n c_{S K_{i}^{\prime}}\left[E n c_{x}\left[X_{i} \| F_{i}\right]\right], F_{i}, I D_{i}, T_{i}^{d}\right\rangle$ to $A S$, where $T_{i}^{d}$ is the current timestamp.

6) After receiving the message at timestamp $T_{s}^{d}, A S$ checks the format of $I D_{i}$ and $\left(T_{s}^{d}-T_{i}^{d}\right) \leq \Delta T$. If either is invalid $A S \quad$ rejects the message;
$\left(X_{i}^{\prime} \| F_{i}^{\prime}\right)=D e c_{x}\left[\operatorname{Dec}_{S K_{i}}\left[E n c_{S K_{i}^{\prime}}\left[E n c_{x}\left[X_{i} \| F_{i}\right]\right]\right]\right]$
$\left\langle E n c_{S K_{i}}\left[X_{i}^{\prime}\right], H_{i}\left(=h\left(x \| F_{i}\right) \oplus S K_{i}\right), T_{s}^{d}\right\rangle$ to $U_{i}$.

7) After receiving $\left\langle E n_{S K_{i}}\left[X_{i}^{\prime}\right], H_{i}\left(=h\left(x \| F_{i}\right) \oplus S K_{i}\right), T_{s}^{d}\right\rangle$ at timestamp $T_{j}^{d}, U_{i} \operatorname{checks}\left(T_{s}^{d}-T_{j}^{d}\right) \leq \Delta T$. If false $U_{i}$ rejects the message, otherwise $U_{i}$ obtains the plaintext of the file by decoding file by computing

$$
\text { Dec_File }=\left[\begin{array}{l}
\operatorname{Dec}_{h\left(I D_{i} \| \psi_{i}\right)}\left[\operatorname{Dec}_{S K_{i}^{\prime}}\left[\operatorname{Enc}_{S K_{i}}\left[X_{i}\right]\right]\right] \\
\| \operatorname{Dec}_{h\left(x \| F_{i}\right)}\left[\operatorname{Enc}_{h\left(x \| F_{i}\right)}\left[\text { File }_{(n-r) b i t s}\right]\right]
\end{array}\right]
$$

\section{Authentication with File Decryption Phase in a Different Session}

When $U_{i}$ wants to decrypt the stored encrypted file from the USB MSD in a different session, the following steps are executed between $U_{i}$ and $A S$ :

1) After checking the provided password $p w_{i}$, identity $I D_{i}$ and biometric parameter $B_{i}^{\prime}$ of $U_{i}$ as described in IV.B, the USB MSD generates random number $\delta_{i}$ and then computes $\hat{W}_{i}=h\left(L_{i}\left\|I D_{i}\right\| \delta_{i}\left\|F_{i}\right\| \hat{T}_{i}\right)$, $\hat{Z}_{i}=L_{i} \oplus \delta_{i}$ and $\hat{Q}_{i}=E N C_{x}\left[X_{i} \| F_{i}\right] \oplus \delta_{i}$, where $F_{i}$ and $\hat{T}_{i}$ are the indexes of the file and the current timestamp of $U_{i}$ respectively. Message $M S G 3=\left\langle I D_{i}, F_{i}, \hat{Q}_{i}, \hat{Z}_{i}, \hat{W}_{i}, \hat{T}_{i}\right\rangle$ is sent to $A S$.

2) After receiving $M S G 3$ at timestamp $\hat{T}_{s}, A S$ checks the format of $I D_{i}$ and $\left(\hat{T}_{s}-\hat{T}_{i}\right) \leq \Delta T$. If either are invalid $A S$ rejects $M S G 3$; otherwise $A S$ computes $L_{i}^{\prime}=h\left(I D_{i} \| x\right)$ and $\delta_{i}^{\prime}=L_{i}^{\prime} \oplus \hat{Z}_{i}$. Then $A S$ checks $\hat{W}_{i}=h\left(L_{i}^{\prime}\left\|I D_{i}\right\| \delta_{i}^{\prime}\left\|F_{i}\right\| \hat{T}_{i}\right)$. If the equality fails $A S$ rejects $M S G 3$; otherwise $A S$ generates a random number $\gamma_{i}$ and computes $\left(X_{i}^{\prime} \| F_{i}^{\prime}\right)=D E C_{x}\left[\hat{Q}_{i} \oplus L_{i}^{\prime}\right]$. Then $A S$ checks $F_{i}=F_{i}^{\prime}$. If true, $A S$ further computes $\quad \hat{Y}_{i}=\gamma_{i} \oplus \dot{L}_{i}^{\prime} \quad, \quad S \hat{K}_{i}=h\left(\delta_{i}^{\prime}\left\|\gamma_{i}\right\| L_{i}^{\prime} \| \hat{T}_{S}\right) \quad, \quad H_{i}=h\left(x \| F_{i}\right) \oplus S \hat{K}_{i} \quad, \quad \hat{E}_{i}=X_{i}^{\prime} \oplus S \hat{K}_{i} \quad$ and $\hat{D}_{i}=h\left(I D_{i}\left\|\gamma_{i}\right\| \delta_{i}^{\prime}\left\|\hat{T}_{S}\right\| S \hat{K}_{i}\left\|h\left(x \| F_{i}\right)\right\| X_{i}^{\prime}\right) . A S$ then sends message $M S G 4=\left\langle\hat{E}_{i}, \hat{D}_{i}, \hat{Y}_{i}, H_{i}, \hat{T}_{s}\right\rangle$ to $U_{i}$.

3) After receiving $M S G 4$ at timestamp $\hat{T}_{j}, U_{i}$ checks $\left(\hat{T}_{j}-\hat{T}_{s}\right) \leq \Delta T$. If false, $U_{i}$ rejects message $M S G 4$; otherwise $U_{i}$ computes $\gamma_{i}^{\prime}=\hat{Y}_{i} \oplus L_{i}, S \hat{K}_{i}^{\prime}=h\left(\delta_{i}\left\|\gamma_{i}^{\prime}\right\| L_{i} \| \hat{T}_{S}\right),\left[h\left(x \| F_{i}\right)\right]^{\prime}=H_{i} \oplus S \hat{K}_{i}^{\prime}, \hat{X}_{i}^{\prime}=\hat{E}_{i} \oplus S \hat{K}_{i}^{\prime}$ and checks equality $\hat{D}_{i}=h\left(I D_{i}\left\|\gamma_{i}^{\prime}\right\| \delta_{i}\left\|\hat{T}_{s}\right\| S \hat{K}_{i}^{\prime}\left\|\left[h\left(x \| F_{i}\right)\right]\right\| \hat{X}_{i}^{\prime}\right)$. If it fails $U_{i}$ rejects message $M S G 4$; 
otherwise $U_{i}$ decrypts $\hat{X}_{i}^{\prime}$ by using the key $h\left(I D_{i} \| \psi_{i}\right)$ to recover the plaintext of the file as File $_{\text {rbits }}=D E C_{h\left(I D_{i} \| \psi_{i}\right)}\left[\hat{X}_{i}^{\prime}\right]$ and also decrypts $\operatorname{ENC}_{h\left(x \| F_{i}\right)}\left[\right.$ File $\left._{(n-r) b i t s}\right]$ by using the key $\left[h\left(x \| F_{i}\right)\right]$ as File $_{(n-r) b i t s}=\operatorname{Dec}_{h\left(x \| F_{i}\right)}\left[\operatorname{Enc}_{h\left(x \| F_{i}\right)}\left[\right.\right.$ File $\left.\left._{(n-r) b i t s}\right]\right]$, where File $=\left(\right.$ File $_{\text {rbits }} \|$ File $\left._{(n-r) b i t s}\right)$.

\section{Password Update Phase}

When a user $U_{i}$ wants to change their password, the password update phase is invoked.

1) $U_{i}$ inserts their USB MSD into the client USB port and inputs their current password $p w_{i}$, identity $I D_{i}$, biometric parameter $B_{i}^{\prime}$ and their new password $p w_{i}^{[n e w]}$. The USB MSD computes $B_{i}=G_{i} \oplus h\left(I D_{i} \| p w_{i}\right)$ and checks $\operatorname{des}\left(B_{i}, B_{i}^{\prime}\right) \leq d$. If it fails the MSD rejects $U_{i}$; otherwise the device computes $\theta_{i}=K_{i} \oplus h\left(I D_{i} \| p w_{i}\right), \psi_{i}=\operatorname{Rep}\left(B_{i}, \theta_{i}\right)$ and $L_{i}\left(=h\left(I D_{i} \| x\right)\right)=A_{i} \oplus h\left(p w_{i} \| \psi_{i}\right)$. Then it checks $C_{i}=h\left(L_{i} \| h\left(p w_{i} \| \psi_{i}\right)\right)$. If the equality fails then the device rejects $U_{i}$; otherwise the USB MSD executes the next step.

2) Then the USB MSD computes $A_{i}^{[\text {new }]}=L_{i} \oplus h\left(p w_{i}^{[n e w]} \| \psi_{i}\right), \quad C_{i}^{[\text {new }]}=h\left(L_{i} \| h\left(p w_{i}^{[n e w]} \| \psi_{i}\right)\right)$, $G_{i}^{[n e w]}=B_{i} \oplus h\left(I D_{i} \| p w_{i}^{[n e w]}\right)$ and $K_{i}^{[n e w]}=\theta_{i} \oplus h\left(I D_{i} \| p w_{i}^{[n e w]}\right)$. The MSD then replaces $\left\langle A_{i}, C_{i}, G_{i}, K_{i}\right\rangle$ with $\left\langle A_{i}^{[\text {new }]}, C_{i}^{[\text {new }]}, G_{i}^{[n e w]}, K_{i}^{[n e w]}\right\rangle$ respectively.

\section{Security Analysis of Proposed Scheme}

The formal security analysis of the proposed scheme under the random oracle model is presented in this section. This security analysis uses the formal security analysis under the generic group model of cryptography. In the following, this work defines random oracles for the formal security analysis of the proposed scheme:

- Oracle $\mathcal{H}$ is a random oracle which maintains a tuple $\langle y, m\rangle$ such that $y=h(m)$. It returns $m$ from $y$ upon receiving a query $(q H, y)$ if $\langle y, m\rangle$ is present in the tuple; otherwise returns a random number $r_{1}$. Then it stores a new entry $\left\langle y, r_{1}\right\rangle$ into its tuple.

- Oracle FE is a random oracle which contains two parts:

1. OracleFE $E_{\mathrm{GEN}}$ unconditionally outputs the pair $(\psi, \theta)$ from the corresponding tuple $\langle b, \theta, \psi\rangle$ upon receiving a query $(q G E N, b)$ such that $(\psi, \theta) \leftarrow G E N(b)$ if $\langle b, \theta, \psi\rangle$ is present in its tuple; otherwise returns two random numbers $r_{2}$ and $r_{3}$. Then it stores new entry $\left\langle b, r_{2}, r_{3}\right\rangle$ into its tuple.

2. Oracle $F E_{\mathrm{REP}}$ unconditionally outputs $\psi$ from the corresponding tuple $\left\langle b^{\prime}, \theta, \psi\right\rangle$ upon receiving a query $\left(q R E P, b^{\prime}, \theta\right)$ such that $\psi \leftarrow R E P\left(b^{\prime}, \theta\right)$ if $\left\langle b^{\prime}, \theta, \psi\right\rangle$ is present in its tuple; otherwise returns random number $r_{4}$. Then it stores new entry $\left\langle b^{\prime}, \theta, r_{4}\right\rangle$ into its tuple. 
- OracleSK is a random oracle which contains two parts:

1. Oracle $S K_{\mathrm{ENC}}$ unconditionally outputs the ciphertext $C$ from its tuple $\langle M, C\rangle$ upon receiving a query $(q E N C, M)$ such that $C=E N C_{k}[M]$ if $\langle M, C\rangle$ is present in its tuple; otherwise returns random number $r_{5}$ from the ciphertext space. It then stores a new entry $\left\langle M, r_{5}\right\rangle$ in its tuple.

2. OracleSK $K_{D \mathrm{EC}}$ unconditionally outputs a key $k$ and plaintext $M$ from its tuple $\langle C, k, M\rangle$ upon receiving a query $(q D E C, C)$ such that $M=E N C_{k}[C]$ if $\langle C, k, M\rangle$ is present in its tuple; otherwise returns two random numbers $r_{6}$ and $r_{7}$ from key space. Then it stores new entry $\left\langle C, r_{6}, r_{7}\right\rangle$ in its tuple.

Theorem 1: Under the assumption that $h(\cdot)$ and $E N C / D E C$ act as random oracles, the proposed scheme derived from this Three-factor Security Protocol (TSP) work is then provably secure against an adversary $A$ for deriving the secret key $x$ of an authentication server $A S$ after obtaining the stored information into the memory of the MSD, and capturing the login message and the reply messages of the authentication plus file encryption plus file retrieval phase during communication between $U_{i}$ and $A S$ in the current session as well as in a different session.

Proof 1: Assume that $A$ has the ability to derive the secret key $x$ of $A S$, and the MSD of $U_{i}$ is lost or stolen. Thus, $A$ can extract the stored parameters $\left\langle A_{i}, C_{i}, K_{i}, G_{i}\right\rangle$ from the memory of the MSD of $U_{i}$ by power monitoring [11], [12]. $A$ also traps the login message $M S G 1$, the reply messages $M S G 2$, $\left\langle E n c_{S K_{i}^{\prime}}\left[E n c_{x}\left[X_{i} \| F_{i}\right]\right], F_{i}, I D_{i}, T_{i}^{d}\right\rangle$ and $\left\langle E n c_{S K_{i}}\left[X_{i}^{\prime}\right], T_{s}^{d}\right\rangle$ of the authentication plus data retrieval phase at timestamp $T_{i}, T_{s}, T_{i}^{d}$ and $T_{s}^{d}$ respectively. $A$ runs the algorithm derived from this work (TSP), EXP1 ${ }_{A, T S P}^{\text {Oracle }}$ to derive the secret key $x$ of $A S$ as given in Algorithm 1. Define the success probability of $\operatorname{EXP1}_{A, T S P}^{\text {Oracle }}$ as:

$$
\operatorname{Succ1}_{A, T S P}^{\text {Oracle }}=\operatorname{Pr}\left[\operatorname{EXP1}_{A, T S P}^{\text {Oracle }}=1\right]
$$

then the advantage is given by:

$$
A d v 1_{A, T S P}^{\text {Oracle }}(t, q H, q D E C)=\max _{A}\left\{\operatorname{Succ1}_{A, T S P}^{\text {Oracle }}\right\}
$$

where the maximum is taken over all $\boldsymbol{A}$ with the execution time $t$, the number of queries $q H$ made to the

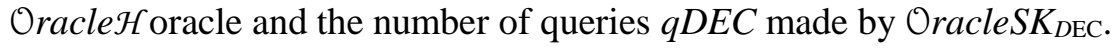

The proposed scheme is said to be provably secure against $A$ deriving the secret key $x$ of $A S$ if $A d v 1_{A, T S P}^{\text {Oracle }}(t, q H, q D E C)<\xi$, for any small $\xi>0$. According to $E X P 1_{A, T S P}^{\text {Oracle }}$, if $A$ is successful in computing the inversion of $h(\cdot)$ as well as extracting the correct secret key, then $\boldsymbol{A}$ can successfully derive the secret key $x$ of $A S$ by using the $O$ racle $\mathcal{H}$ and $O$ racleS $K_{D E C}$ random oracles respectively. But, according to Definition 1 and $3, A d v 1_{A, T S P}^{\text {Oracle }}(t, q H, q D E C) \leq \xi_{1}$, for any small $\xi_{1}>0$. Since the advantage $A d v 1_{A, T S P}^{\text {Oracle }}(t, q H, q D E C)<\xi$, for any small $\xi>0$ because the proposed scheme depends on $A d v 1_{A}^{H}(t)$ and $A d v 1_{A}^{D E C}(t)$. Thus, this proposed scheme is secure against $\boldsymbol{A}$ for deriving the secret key $x$ of $S$. 


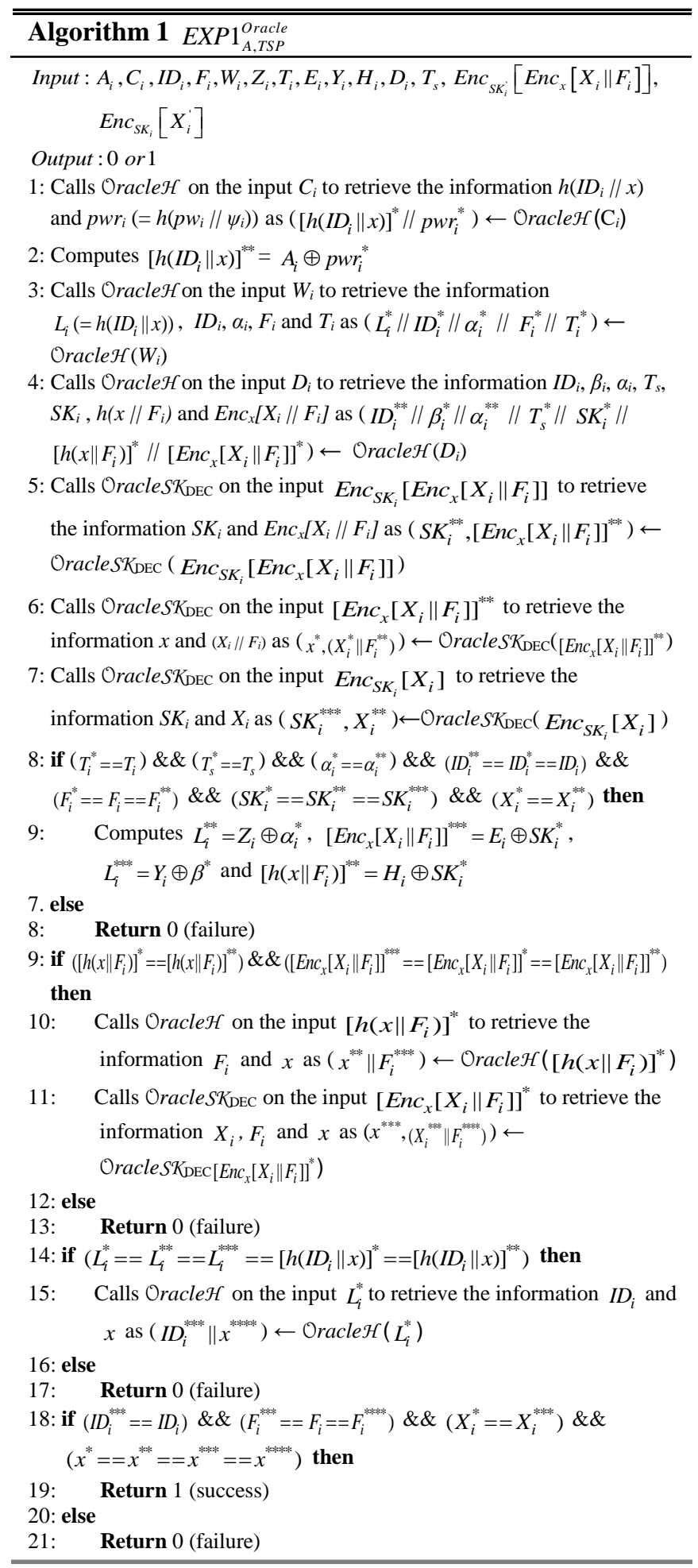


Theorem 2: Under the assumption that $h(\cdot)$ and $F E$ act as random oracles, the proposed Three-factor Security Protocol (TSP) scheme derived from this work is provably secure against adversary $\boldsymbol{A}$ for deriving the password $p w_{i}$ of $U_{i}$ after obtaining the stored information in the MSD, and capturing the login message and reply message of the authentication plus file encryption plus file retrieval phase during communication between $U_{i}$ and $A S$ in the current session as well as in any other session.

Proof 2: Apply the same assumptions as described in Theorem 1. There is no chance to derive password $p w_{i}$ of $U_{i}$ from communication messages because they are independent of the password. Then $A$ runs the algorithm derived from this work (TSP), EXP2 ${ }_{A, T S P}^{\text {Oracle }}$ to derive the password $p w_{i}$ of $U_{i}$ as given in Algorithm 2. Define the success probability of $\operatorname{EXP}_{A, T S P}^{\text {Oracle }}$ as:

$$
\operatorname{Succ2}{ }_{A, T S P}^{\text {Oracle }}=\operatorname{Pr}\left[\operatorname{EXP2}_{A, T S P}^{\text {Oracle }}=1\right]
$$

then the advantage is given by:

$$
A d v 2_{A, T S P}^{\text {Oracle }}(t, q H, q F E)=\max _{A}\left\{\operatorname{Succ} 2_{A, T S P}^{\text {Oracle }}\right\}
$$

where the maximum is taken over all $A$ with the execution time $t$, the number of queries $q H$ made to the Oracle $\mathcal{H}$ oracle and the number of queries $q F E$ made by $O$ racle $F E_{\text {REP. }}$. Again, the proposed scheme is said to be provably secure against $A$ deriving the password $p w_{i}$ of $U_{i}$ if $A d v 2_{A, T S P}^{\text {Oracle }}(t, q H, q F E)<\xi$, for any small $\xi>0$ . According to EXP2 ${ }_{A, T S P}^{\text {Oracle }}$, if $A$ is successful in computing the inversion of $h(\cdot)$ as well as extract the correct derived biometric parameter $\psi_{i}$, they can successfully derive the password $p w_{i}$ of $U_{i}$ by using of the OracleH and $O$ racle $F E_{\mathrm{REP}}$ random oracles respectively. But, according to Definition 1 and 2 , $A d v 2_{A, T S P}^{\text {Oracle }}(t, q H, q F E) \leq \xi_{1}$ , for any small $\xi_{1}>0$. Since, the advantage $A d v 2_{\text {A.TSP }}^{\text {Oracle }}(t, q H, q F E) \leq \xi$, for any small $\xi>0$ because the proposed scheme depends on $A d v 2_{A}^{H}(t)$ and $A d v 1_{A}^{F E}(t)$. Thus, this proposed scheme is secure against $A$ for deriving the password $p w_{i}$ of $U_{i}$. 


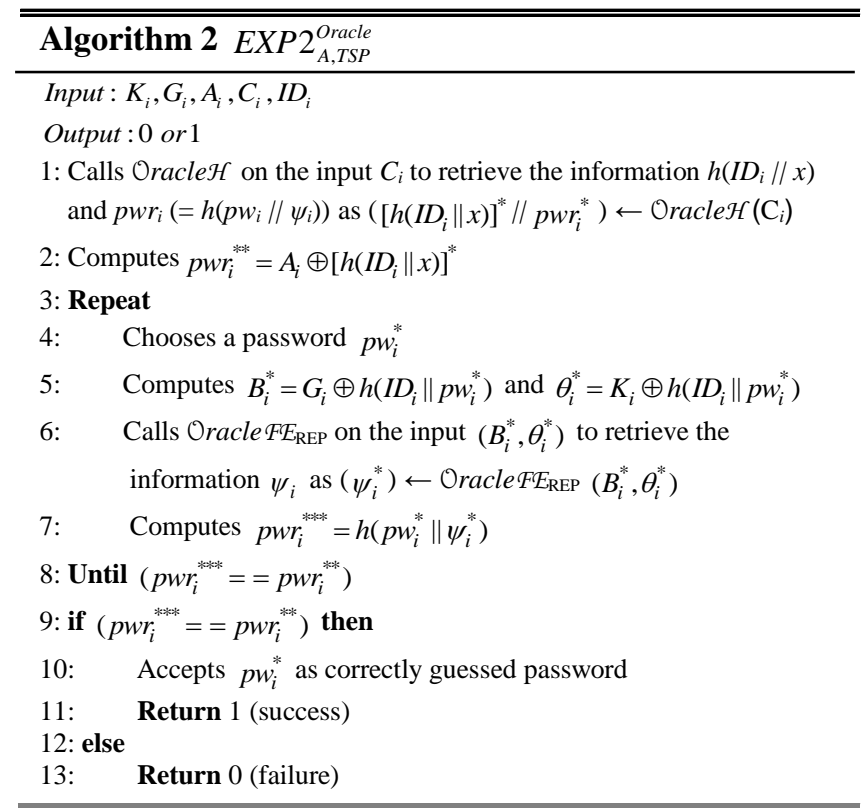

Theorem 3: Under the assumption that $h(\cdot)$ and ENC/DEC act as random oracles, then the Three-factor Security Protocol (TSP) scheme derived from this work is provably secure against $\boldsymbol{A}$ deriving the shared secret session key $S K$ between $U_{i}$ and $A S$ after getting the stored information into the memory of the MSD device, and trapping the login message and reply message of authentication plus file encryption plus file retrieval phase during communication between $U_{i}$ and $A S$ in the current session as well as any other session.

Proof 3: Apply the same assumptions as described in Theorem 1. Then $A$ runs the algorithm derived from this work (TSP), EXP3 $3_{A, T S P}^{\text {Oracle }}$ to derive the session key $S K_{i}$ between $U_{i}$ and $A S$ as given in Algorithm 3. Define the success probability of $E X P 3_{A, T S P}^{\text {Oracle }}$ as:

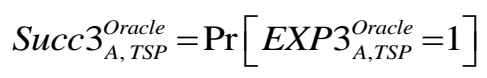

then the advantage is given by:

$$
A d v 3_{A, T S P}^{\text {Oracle }}(t, q H, q D E C)=\max _{A}\left\{\operatorname{Succ} 3_{A, T S P}^{\text {Oracle }}\right\}
$$

where the maximum is taken over all $\boldsymbol{A}$ with the execution time $t$, the number of queries $q H$ made to the


to be provably secure against $A$ deriving the session key $S K_{i}$ between $U_{i}$ and $A S$ if $A d v 3_{A, T S P}^{\text {Oracle }}(t, q H, q D E C) \leq \xi$ , for any small $\xi>0$. According to $E X P 3_{A, T S P}^{\text {Oracle }}$, if $A$ is successful in computing the inversion of $h(\cdot)$ and extracting the correct key, they can successfully derive the shared secret session key $S K_{i}$ between $U_{i}$ and $A S$ by using of the $O$ racleH and racleSK $_{D \mathrm{EC}}$ random oracles respectively. But, according to Definition 1 and $3, A d v 3_{A, T S P}^{\text {Oracle }}(t, q H, q D E C) \leq \xi_{1}$, for any small $\xi_{1}>0$. Since, the advantage $A d v 3_{A, T S P}^{\text {Oracle }}(t, q H, q D E C) \leq \xi$, for any small $\xi>0$ because the proposed scheme depends on $A d v 3_{A}^{H}(t)$ and $A d v 3_{A}^{D E C}(t)$. Thus, the proposed scheme is secure against $\boldsymbol{A}$ deriving the shared secret session key $S K_{i}$ between $U_{i}$ and $A S$. 




\section{A. Discussion of Presented Theorems}

Theorem 2 demonstrated that the proposed scheme is secure against the off-line password guessing attack.

Theorem 3 demonstrates that the proposed scheme is secure against the session key recovery attack, because without knowing random numbers $\alpha, \beta$ (for current session), and $\delta, \gamma$ (for a different session) then $A$ cannot compute the session key $S K$. In the proposed scheme, all communicating messages depend on random numbers and the timestamp. So, all the communication messages are guaranteed to be different for every session. Thus, $A$ cannot mount a replay attack on this proposed scheme.

In this proposed scheme, $A$ cannot mount a forgery attack without knowing secret password $p w_{i}$ of $U_{i}$, the secret key $x$ of the server $A S$ and random numbers generated by $U_{i}$ and $A S$ respectively.

Theorems 1 and 2 show that the secret information of the authentication server and the user are secure from $A$. Thus, it is infeasible to mount a forgery attack on this proposed scheme. 


\section{Performance evaluation of Proposed Scheme}

This section compares the performance of the proposed scheme with related schemes in the literature [1][3], [5], [6]. The login and authentication phases for both file encryption and file decryption of the proposed scheme have been compared with the related existing schemes in the literature [1]-[3], [5], [6] because these phases are commonly used.

Table II presents the communication (overhead) and storage costs of this work compared to the literature. It can be seen that the communication cost of this work is the same for data encryption in the literature, but has a significant advantage in the data decryption within the current session as was the objective of this work. The storage cost of this work is also comparable to the literature.

TABLE II

COMPARISON OF COMMUNICATION AND STORAGE COSTS OF SCHEMES IN THE CURRENT LiTERATURE COMPARED TO THIS PROPOSED SCHEME

\begin{tabular}{|c|c|c|c|c|c|}
\hline \multirow{3}{*}{ Comparison Metric } & \multicolumn{4}{|c|}{ Communication Cost (bits) } & \multirow{3}{*}{$\begin{array}{c}\text { Storage } \\
\text { Cost (bits) }\end{array}$} \\
\hline & \multicolumn{2}{|c|}{ File encryption } & \multicolumn{2}{|c|}{ Authentication + File retrieval } & \\
\hline & Login & Authentication & $\begin{array}{c}\text { (Different session) } \\
\text { Login + Authentication }\end{array}$ & $\begin{array}{c}\text { (Current session) } \\
\text { Login + Authentication }\end{array}$ & \\
\hline $\mathrm{Ku}$ and Chen [1] & 320 & 256 & - & - & 384 \\
\hline Yoon, Ryu and Yoo [2] & 320 & 256 & - & - & 512 \\
\hline Yang, Wu and Chiu [3] & 3328 & 1280 & $4608=(3328+1280)$ & $4608=(3328+1280)$ & 2176 \\
\hline He et al.[6] & 512 & 640 & $1152=(512+640)$ & $1152=(512+640)$ & 384 \\
\hline Proposed scheme & 640 & 768 & $1408=(640+768)$ & $768=(384+384)$ & 640 \\
\hline
\end{tabular}

Table III verifies the types of attacks that are considered, the key management and the mutual authentication that the literature uses compared to this work.

TABLE III

Comparison of AtTack Vulnerability of SCHEMES in the Current Literature COMPAREd TO THIS PROPOSED SCHEME

\begin{tabular}{|c|c|c|c|c|c|c|}
\hline Attack Vulnerability / Feature & $\begin{array}{l}\text { Ku and Chen } \\
{[1]}\end{array}$ & $\begin{array}{l}\text { Yoon, Ryu } \\
\text { and Yoo [2] }\end{array}$ & $\begin{array}{l}\text { Yang, Wu and } \\
\text { Chiu [3] }\end{array}$ & $\begin{array}{l}\text { Lee et al. } \\
{[5]}\end{array}$ & $\begin{array}{l}\text { He et al. } \\
{[6]}\end{array}$ & $\begin{array}{l}\text { Proposed } \\
\text { scheme }\end{array}$ \\
\hline User impersonation attack & yes [2] [14] & yes [14] & no & no & no & no \\
\hline Server masquerading attack & yes [14] & yes [14] & yes [15] & no & no & no \\
\hline Insider attack & no & no & no & no & no & no \\
\hline Off-line password guessing attack & yes [14] & yes [14] & no & yes [6] & no & no \\
\hline Inefficient login phase & yes [14] & yes [14] & yes & no & no & no \\
\hline Denial of service (DoS ) attack & yes [14] & yes [14] & no & yes [6] & no & no \\
\hline Password change phase attack & yes [2] [14] & yes [14] & no & - & - & no \\
\hline Replay attack & no & no & yes & yes [6] & yes & no \\
\hline Session key agreement & no & no & yes & yes & yes & yes \\
\hline Mutual authentication & no & no & yes & no & yes & yes \\
\hline
\end{tabular}

Table IV presents the computational cost of this work compared to the literature. $T_{h}$ is the time required for the hashing operation, $T_{P m}$ for point multiplication operation, $T_{m}$ for scalar multiplication operation, $T_{d i v}$ for division operation and $T_{E n} / T_{D e}$ for the symmetric key encryption/decryption operation. Typically, the time complexity associated with these operations can be expressed as $T_{E n} / T_{D e}=T_{h}>T_{P m}>T_{m} \approx T_{d i v}$ [13]. It can be seen that this work significantly reduces the computation cost for both data encryption and data retrieval as well as authentication. 
It can be assumed that the identity $I D_{i}$, the file index $F_{i}$ and the password $p w_{i}$ are length of 64 bits each, cryptographic one-way hash function $h(\cdot)$ and symmetric key encryption/decryption, random number and timestamp returns 128 bits for each block. Since the communication overhead for the login, file encryption and the authentication phase as well as login, file decryption and authentication in different sessions is $(2 \times 64)+(8 \times 128)+256=1408$ bits, and login, file decryption and authentication in current session phase is $(2 \times 64)+(5 \times 128)=768$ bits. Therefore, this work achieves a much lower communication overhead than the work in the literature [3], [5], [6]. Low communication overhead, low storage cost, low computational cost and resistance of all possible attacks indicate that the proposed scheme provides an efficient security protocol offering a practical solution for enhanced security of mass market USB MSD.

TABLE IV

COMPARISON OF COMPUTATIONAL COST OF RELATED SCHEMES COMPARED TO THIS PROPOSED SCHEME

\begin{tabular}{|c|c|c|c|c|c|c|c|}
\hline & \multicolumn{3}{|c|}{ File encryption } & \multicolumn{4}{|c|}{ Authentication + File retrieval } \\
\hline & \multirow{2}{*}{$\begin{array}{c}\text { Login } \\
\text { (USB MSD) }\end{array}$} & \multicolumn{2}{|c|}{ Authentication } & \multicolumn{2}{|c|}{ Different session } & \multicolumn{2}{|c|}{ Current session } \\
\hline & & (USB MSD) & (Server) & (USB MSD) & (Server) & (USB MSD) & (Server) \\
\hline $\mathrm{Ku}$ and Chen [1] & $2 T_{h}$ & $1 T_{h}$ & $3 T_{h}$ & - & - & - & - \\
\hline Yoon, Ryu and Yoo [2] & $2 T_{h}$ & $1 T_{h}$ & $3 T_{h}$ & - & - & - & - \\
\hline Yang, Wu and Chiu [3] & $\begin{array}{l}1 T_{m}+1 T_{h} \\
+2 T_{e}\end{array}$ & $\begin{array}{l}2 T_{h}+2 T_{e}+ \\
1 T_{D e}+1 T_{E n}\end{array}$ & $\begin{array}{l}1 T_{e n}+1 T_{d i v}+2 \\
T_{m}+3 T_{e}+3 T_{h}\end{array}$ & $\begin{array}{l}1 T_{m}+3 T_{h} \\
+4 T_{e}+2 T_{D e}\end{array}$ & $\begin{array}{l}1 T_{e n}+1 T_{d i v}+2 \\
T_{m}+3 T_{e}+3 T_{h}\end{array}$ & $\begin{array}{l}1 T_{m}+3 T_{h} \\
+4 T_{e}+2 T_{D e}\end{array}$ & $\begin{array}{l}1 T_{e n}+1 T_{d i v}+2 T_{m}+ \\
3 T_{e}+3 T_{h}\end{array}$ \\
\hline Lee et al. [5] & $4 T_{h}+1 T_{P m}$ & $\begin{array}{l}2 T_{h}+1 T_{P m}+ \\
1 T_{E n}+1 T_{D e}\end{array}$ & $\begin{array}{l}4 T_{h}+2 T_{P m} \\
+1 T_{E n}\end{array}$ & $\begin{array}{l}6 T_{h}+2 T_{P m} \\
+2 T_{D e}\end{array}$ & $\begin{array}{l}4 T_{h}+2 T_{P m} \\
+1 T_{E n}\end{array}$ & $\begin{array}{l}6 T_{h}+2 T_{P m} \\
+2 T_{D e}\end{array}$ & $\begin{array}{l}4 T_{h}+2 T_{P m} \\
+1 T_{E n}\end{array}$ \\
\hline He et al. [6] & $4 T_{h}+1 T_{P m}$ & $\begin{array}{l}3 T_{h}+1 T_{P m}+ \\
1 T_{E n}+1 T_{D e}\end{array}$ & $\begin{array}{l}5 T_{h}+2 T_{P m} \\
+1 T_{E n}\end{array}$ & $\begin{array}{l}7 T_{h}+2 T_{P m} \\
+2 T_{D e}\end{array}$ & $\begin{array}{l}5 T_{h}+2 T_{P m} \\
+1 T_{E n}\end{array}$ & $\begin{array}{l}7 T_{h}+2 T_{P m} \\
+2 T_{D e}\end{array}$ & $\begin{array}{l}5 T_{h}+2 T_{P m} \\
+1 T_{E n}\end{array}$ \\
\hline Proposed scheme & $5 T_{h}+1 T_{E n}$ & $2 T_{h}$ & $4 T_{h}+1 T_{E n}$ & $7 T_{h}+1 T_{D e}$ & $4 T_{h}+1 T_{D e}$ & $1 T_{E n}+2 T_{D e}$ & $2 T_{D e}+1 T_{E n}$ \\
\hline
\end{tabular}

\section{Conclusion}

An efficient mutual authentication protocol has been presented to encrypt files in a Universal Serial Bus (USB) Mass Storage Device (MSD) enabling secure and usable "USB memory sticks." This paper has contributed a novel concept to the current state of the art in biometric security algorithms by defending against security attacks and improving device usability across different sessions. Moreover, the paper has formally proved that the proposed protocol can withstand relevant security weaknesses. A performance comparison has also been made with the literature to confirm that the proposed scheme achieves a significantly lower computation cost and communication cost than other related schemes. The overall efficiency demonstrates that USB based MSDs with biometric security sensors can be implemented in order to provide significant security and usability for the consumer and beyond. 


\section{REFERENCES}

[1] W.-C. Ku, and S.-M. Chen, "Weaknesses and improvements of an efficient password based remote user authentication scheme using smart cards," IEEE Trans. Consumer Electron., vol. 50, no. 1, pp. 204-207, Feb. 2004.

[2] E.-J. Yoon, E.-K. Ryu, and K.-Y. Yoo, "Further improvement of an efficient password based remote user authentication scheme using smart cards," IEEE Trans. Consumer Electron., vol. 50, no. 2, pp. 612-614, May 2004.

[3] F.-Y. Yang, T.-D. Wu, and S.-H. Chiu, "A secure control protocol for USB mass storage devices," IEEE Trans. Consumer Electron., vol. 56, no. 4, pp. 2339-2343, Nov. 2010.

[4] D.-J. Kim, and K.-S. Hong, "Multimodal biometric authentication using teeth image and voice in mobile environment," IEEE Trans. Consumer Electron., vol. 54, no. 4, pp. 1790-1797, Nov. 2008.

[5] C. Lee, C. Chen, and P. Wu, "Three-factor control protocol based on elliptic curve cryptosystem for universal serial bus mass storage devices," IET Computers \& Digital Techniques, vol. 7, no. 1, pp. 4855, Jan. 2013.

[6] D. He, N. Kumar, J.-H. Lee, and R. S. Sherratt, "Enhanced three-factor security protocol for consumer USB mass storage devices," IEEE Trans. Consumer Electron., vol. 60, no. 1, pp. 30-37, Feb. 2014.

[7] D. Giri, R. S. Sherratt, T. Maitra, and R. Amin, "Efficient Biometric and Password Based Mutual Authentication for Consumer USB Mass Storage Devices," IEEE Trans. Consumer Electron., vol. 61, no. 4, pp. 491-499, Nov. 2015.

[8] Y. Dodis, L. Reyzin, and A. Smith, "Fuzzy extractors: How to generate strong keys from biometrics and other noisy data," in Proc. Int. Conf. Theory and Applications of Cryptographic Techniques, Interlaken, Switzerland, LNCS, Vol. 3027, pp 523-540, 2004.

[9] B. A. Forouzan and D. Mukhopadhyay, Cryptography and Network Security 2/e. Tata-McGraw Hill, TMH, 2nd edition, 2010.

[10] N. Ferguson, B. Schneier, and T. Kohno, Cryptography Engineering Design Principles and Practical Applications, John Wiley \& Sons, Mar. 2010.

[11] P. C. Kocher, J. Jaffe, and B. Jun, "Differential power analysis," in Proc. 19th Annual Int. Cryptology Conf. Advances in Cryptology, Santa Barbara, CA, pp 388-397, Aug. 1999.

[12] T. S. Messerges, E. A. Dabbish, and R. H. Sloan, "Examining smart-card security under the threat of power analysis attacks," IEEE Trans. Comput., vol. 51, no. 5, pp. 541-552, May 2002.

[13] N. R. Potlapally, S. Ravi, A. Raghunathan, and N. K. Jha, "A study of the energy consumption characteristics of cryptographic algorithms and security protocols," IEEE Trans. Mobile Comput., vol. 5, no. 2, pp. 128-143, Feb. 2006.

[14] X.-M. Wang, W.-F. Zhang, J.-S. Zhang, and M. K. Khan, "Cryptanalysis and improvement on two efficient remote user authentication scheme using smart card," Computer Standards \& Interfaces, vol. 29, no. 5, pp. 507-512, Jul. 2007.

[15] M. H. Eldefrawy, M. K. Khan, and H. Elkamchouchi, "The use of two authentication factors to enhance the security of mass storage device," in Proc. 11th Int. Conf. Information Technology: New Generations, Las Vegas, NV, pp. 196-200, Apr. 2014. 


\section{BIOGRAPHIES}



Debasis Giri received the Ph.D degree from the Indian Institute of Technology, Kharagpur, India in 2009. He did his masters (M.Tech and M.Sc) both from Indian Institute of Technology, Kharagpur in 2001 and 1998 respectively. Presently he is a Professor in the Department of Computer Science and Engineering, Haldia Institute of Technology, India. He has tenth All India Rank with percentile score 98.42 in the Graduate Aptitude Test in Engineering (GATE) Examination in 1999. His current research interests include cryptography, Network security, Security in Wireless Sensor Networks and Security in VANETs.

Dr. Giri is an Editorial Board Member and a Reviewer of many reputed International Journals. Presently he is an Associate Editor of the Journal of Security and Communication Networks (Wiley), and the Journal of Electrical and Computer Engineering Innovations. He is also a Program Committee member for many International Conferences.



R. Simon Sherratt (M'97-SM'02-F'12) received the B.Eng. degree in Electronic Systems and Control Engineering from Sheffield City Polytechnic, UK in 1992, M.Sc. in Data Telecommunications in 1994 and Ph.D. in video signal processing in 1996 from the University of Salford, UK.

In 1996, he has appointed as a Lecturer in Electronic Engineering at the University of Reading where he is now Professor of Biosensors. His research topic is signal processing and communications in consumer devices focusing on wearable devices and healthcare. He received the $1^{\text {st }}$ place IEEE Chester Sall Memorial Award in 2006 and $2^{\text {nd }}$ place in 2016. He is a reviewer for the IEEE SENSORS JOURNAL is now the Editor-in-Chief of the IEEE TRANSACTIONS ON CONSUMER ELECTRONICS.



Tanmoy Maitra received his B.E. degree in Computer Science and Engineering from Burdwan University, India in 2009 and his M.Tech degree in Computer Science and Engineering from WBUT, India in 2013. Currently, he is pursuing a Ph.D from Jadavpur University, India. His research interests include wireless sensor networks and applied cryptology. 